\title{
Targeted capture and sequencing of gene-sized DNA molecules
}

Michael Giolai ${ }^{1}$, Pirita Paajanen ${ }^{1}$, Walter Verweij ${ }^{1}$, Lawrence Percival-Alwyn¹, David Baker ${ }^{1}$, Kamil Witek$^{2}$, Florian Jupe ${ }^{2,3}$, Glenn Bryan4, Ingo Hein ${ }^{4}$, Jonathan D. G. Jones ${ }^{2}$, and Matthew D. Clark ${ }^{1,5}$ ${ }^{1}$ Earlham Institute (EI), Norwich Research Park, Norwich, UK, ${ }^{2}$ The Sainsbury Laboratory, Norwich Research Park, Norwich, UK, ${ }^{3}$ Plant Biology Laboratory, Salk Institute for Biological Studies, La Jolla, CA, ${ }^{4}$ The James Hutton Institute, Dundee, UK, and ${ }^{5}$ School of Environmental Sciences, University of East Anglia, Norwich Research Park, Norwich, UK

\author{
BioTechniques 61:315-322 (December 2016) doi 10.2144/000114484 \\ Keywords: targeted capture; gene enrichment; PacBio; RenSeq; NB-LRR gene; resistance gene
}

Supplementary material for this article is available at www.BioTechniques.com/article/114484.

Targeted capture provides an efficient and sensitive means for sequencing specific genomic regions in a high-throughput manner. To date, this method has mostly been used to capture exons from the genome (the exome) using short insert libraries and short-read sequencing technology, enabling the identification of genetic variants or new members of large gene families. Sequencing larger molecules results in the capture of whole genes, including intronic and intergenic sequences that are typically more polymorphic and allow the resolution of the gene structure of homologous genes, which are often clustered together on the chromosome. Here, we describe an improved method for the capture and single-molecule sequencing of DNA molecules as large as $7 \mathrm{~kb}$ by means of size selection and optimized PCR conditions. Our approach can be used to capture, sequence, and distinguish between similar members of the NB-LRR gene family-key genes in plant immune systems.

The plant innate immune system uses complex networks to recognize the presence of potential intruders and protect the plant against disease development. Pathogen-derived molecules, the so-called pathogen-associated molecular patterns (PAMPs), are recognized by plant pattern recognition receptors (PRRs) on a plant cell's surface and initiate an immune response that is often sufficient to stop the pathogen from further growth. However, effector proteins released by adapted pathogens can weaken this resistance; thus, plants have a second layer of defense in the form of nucleotide binding-site leucine- rich repeat (NB-LRR) genes that directly or indirectly interfere with the effector proteins, causing local cell death to prevent further disease development (1). As NB-LRR genes are often the genetic basis of disease resistance in plants, they are also known as resistance genes or R-genes. RenSeq (R-gene enrichment sequencing) has been recently described using shortread sequencing technology $(2,3,4)$. As R-genes often appear in clusters of nearly identical sequences, it is problematic to distinguish highly similar sequences (e.g., recently duplicated genes), including inter- and intragenic regions in such clusters. Here, we demonstrate that the use of RenSeq on DNA fragments up to 7 -kb long in combination with PacBio sequencing results in full contig assemblies covering entire R-gene clusters with inter- and intragenic regions (Figure 1).

\section{Material and methods}

Concentration assessment and quality control

All DNA samples, if not stated otherwise, were quantified using a Qubit 2.0 Fluorometer (Thermo Fisher, Cambridge, UK). DNA fragment sizes

\section{METHOD SUMMARY}

To improve PacBio sequencing of large DNA insert molecules, we developed a strategy to increase the overall size of the targeted DNA molecules by efficiently removing small DNA molecules that are preferentially sequenced on the PacBio platform. The key improvements offered by our method include the use of (i) the Diagenode Megaruptor DNA shearing device to generate a tight distribution of large DNA fragments; (ii) the Sage Scientific Electrophoretic Lateral Fractionator for DNA fragment size selection; and (iii) Kapa HiFi HotStart polymerase to significantly improve amplification yields. The latest PacBio P6-C4 chemistry with an average read length of 10-15 kb allowed us to generate consensus reads with high accuracy. 
were measured using an Agilent Bioanalyzer DNA 12000 chip (Agilent, Stockport, UK).

\section{Genomic DNA extraction}

Genomic DNA was extracted using cetyltrimethylammonium bromide (CTAB lysis), phenol-chloroform extraction, and Qiagen MagAttract HMW DNA Kit (QIAGEN, Manchester, UK) purification. Young frozen Solanum verrucosum (an inbred genotype from accession VER54) leaves (5 g) were ground to a fine powder using a liquid nitrogen-cooled mortar and pestle, distributed between two 50-mL tubes, mixed with $20 \mathrm{~mL}$ CTAB buffer $[100 \mathrm{mM}$ Tris-HCl pH 8.0, 2\% (w/v) CTAB, $1.4 \mathrm{M} \mathrm{NaCl}, 20 \mathrm{mM}$ EDTA] containing $20 \mu \mathrm{g} / \mathrm{mL}$ proteinase $\mathrm{K}$, and incubated at $55^{\circ} \mathrm{C}$ for $20 \mathrm{~min}$. Next, 0.5 volumes ( $8 \mathrm{~mL}$ ) chloroform was added and carefully mixed by inversion $15 x$, followed by centrifugation at $2990 \times$ $g$ in an Eppendorf Centrifuge $5810 \mathrm{R}$ (Eppendorf, Stevenage, UK) for $30 \mathrm{~min}$. The aqueous phase was carefully transferred to a new tube to which 1 volume phenol/chloroform/isoamyl alcohol was added, followed by centrifugation for 30 $\min$ at $2990 \times g$. The aqueous phase was ethanol precipitated by addition of $3 \mathrm{M}$ sodium acetate $(0.1 \times$ DNA volume $)$ $\mathrm{pH} 5.2$ and 2.5 volumes ethanol, mixed, and precipitated at $2990 \times g$ at $4^{\circ} \mathrm{C}$. DNA pellets were washed with ice-cold $70 \%$ ethanol, air-dried, and resuspended in $350 \mu \mathrm{L} 1 \times$ TE buffer (10 mM Tris-HCl, 1 mM EDTA, pH 8.0) containing $10 \cup$ RNase One (Promega, Southampton, UK). The DNA was dissolved overnight at $4^{\circ} \mathrm{C}$ with occasional mixing by inversion. Finally, we purified the DNA with the QIAGEN MagAttract HMW DNA Kit with minor changes to the manufacturer's instructions: we added $150 \mu \mathrm{L} \mathrm{AL}$ Buffer to $200 \mu \mathrm{L}$ DNA (10 $\mu \mathrm{g}$ in TE) and $15 \mu \mathrm{L}$ magnetic bead suspension. From this point, we followed the MagAttract HMW DNA Kit Quick-Start protocol (www.qiagen.com/us/resources/ resourcedetail?id=50e33eab-4f51-490a9dc4-0c3a3469e1d2\&lang=en), and we eluted the DNA in $200 \mu \mathrm{L} 1 \times$ TE buffer.

\section{DNA fragmentation}

We used the Diagenode Megaruptor (Diagenode, Seraing, Belgium) to fragment $10 \mu \mathrm{g}$ of DNA to 8-kb fragments with the following settings: $200-\mu \mathrm{L}$ input volume, 8-kb shearing size, short (a)

(b)

(c)

(d) *

(e)

(f) *

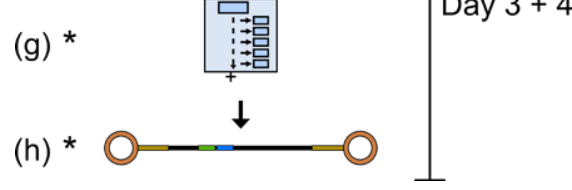

hydropore, pre-load hydropore, wash syringe before shearing.

\section{PreCR repair}

The sheared DNA samples were repaired using the PreCR Repair Mix (New England Biolabs, Hitchin, UK) in a $100 \mu \mathrm{L}$ reaction volume containing 1 $\mu \mathrm{g}$ DNA, 1× ThermoPol Buffer, $1 \times \mathrm{NAD}^{+}$, $0.1 \mathrm{mM}$ dNTPs, and $2 \mu \mathrm{L}$ enzyme mix. The reaction was incubated for $30 \mathrm{~min}$ at $37^{\circ} \mathrm{C}$ followed by a $0.4 \times$ AMPure XP bead (Beckman Coulter, Brea, CA) purification and elution in $60 \mu \mathrm{L} 1 \times$ TE buffer.

Illumina TruSeq DNA library preparation Large insert libraries were constructed using the Illumina TruSeq Low Sample protocol (TruSeq DNA Sample Prep Guide 15026486 C): sheared DNA was end repaired, A-tailed, and Illumina adapters were ligated to both ends of the DNA strands to serve as primer binding sites for subsequent amplification. For all purification steps, a 0.4× AMPure $\mathrm{XP}$ bead clean-up was used to remove smaller DNA fragments. After the ligation termination step, two $0.4 \times$ AMPure XP purifications were performed.

TruSeq library amplification

$\mathrm{PCR}$ reactions were conducted in a G-Storm GS1 (G-Storm, Somerton, UK) thermal cycler. The reaction set-up was as follows: 100 ng Illumina TruSeq library, $0.3 \mu \mathrm{M}$ Illumina Primer Mix, and
Figure 1. The improved R-gene enrichment sequencing (RenSeq) protocol. The proto$\mathrm{col}$ is based on the construction of an Illumina TruSeq library $(a-c)$, size selection of the amplified library (d), RNA bait capture and on bead amplification of the captured library (e-f), a final size selection step (g), and PacBio library construction (h). Altered or improved steps are marked with an asterisk. To construct the TruSeq library, DNA has to be fragmented (a). The fragmented DNA is end-repaired, A-tailed, and Illumina Y-shaped adapters are ligated to both DNA ends (b). The Y-adapters serve as primer binding sites for a first amplification step (c). The PCR-amplified library is size selected on the SageELF (d). The R-genes are captured in an overnight reaction using biotinylated RNA baits designed to bind to R-gene motifs (e). To separate the captured fragments from the non-captured library, a magnetic bead pulldown is performed ( $f$ ). To retrieve and replicate the captured $\mathrm{R}$-gene sequences, the library is amplified on the beads ( $f$ ). Another size-selection step $(g)$ is performed on the amplified library prior to PacBio library assembly and sequencing (h).

25 uL 2x Kapa HiFi HotStart ReadyMix (Kapa Biosystems, London, UK) in a total volume of $50 \mu \mathrm{L}$. We used the following PCR program: $94^{\circ} \mathrm{C}$ for $3 \mathrm{~min}, 10 \times\left[94^{\circ} \mathrm{C}\right.$ $30 \mathrm{~s}, 60^{\circ} \mathrm{C} 30 \mathrm{~s}, 68^{\circ} \mathrm{C} 4 \mathrm{~min}$ ], $68^{\circ} \mathrm{C} 10$ min. The ramp rate was set to $3.0^{\circ} \mathrm{C} / \mathrm{s}$. We obtained $6.1 \mu \mathrm{g}$ of amplified library from the PreCR-treated and $9.0 \mu \mathrm{g}$ from the non-PreCR-repaired sample.

\section{Size selection of the}

Illumina TruSeq library

Before size selection with the Sage Scientific Electrophoretic Lateral Fractionator (SageELF; Sage Science, Beverly, MA), we concentrated the amplified TruSeq libraries to $30 \mu \mathrm{L}$ using precipitation with $0.4 \times$ AMPure XP beads. The amplified TruSeq library $(5.0 \mu \mathrm{g})$ was loaded on a $0.75 \%$ SageELF agarose gel cassette using the following settings: timed separation mode, separation time: $2 \mathrm{~h}$ $15 \mathrm{~min}$. For each library, we pooled and quantified the fractions with the correct size distribution.

Target enrichment using the MYbaits target enrichment kit We vacuum dried (Eppendorf SpeedVac Concentrator 5301; Eppendorf, Arlington, UK) $250 \mathrm{ng}$ of each sizeselected 4-kb, 5-kb, and 7-kb library at $48^{\circ} \mathrm{C}$ for $5 \mathrm{~min}$ and dissolved the DNA in $1.7 \mu \mathrm{L}$ nuclease-free water. The hybridization of DNA with RNA baits was performed according to the MYbaits 
protocol (MYbaits User Manual version 1.3.8) and kit (MYcroarray, Ann Arbor, MI) but with minor changes and in half of the recommended reaction volume. The baits were designed as described by Jupe et al. (3). In brief, the entire bait library was composed of 20,000 unique 120-mer RNA probes designed for the coding regions of potato R-gene sequences between exon-exon boundaries starting from the first nucleotide following the predicted coding region of the R-gene. Sequences were at least 2 times tiled with a 60 nucleotide overlap $(3,5)$. A full list of bait sequences can be found in the SMRT RenSeq protocol (www.nature.com/protocolexchange/ protocols/4663). The Library Master Mix was prepared as follows: $1.7 \mu \mathrm{L}$ resuspended TruSeq library was mixed with $5 \mu \mathrm{L}$ SeqCap plant capture enhancer (Roche, Burgess Hill, UK) and 0.6 Block \#3. The Hybridization Master Mix was prepared by mixing $10 \mu \mathrm{L}$ Hyb \#1, 0.4 $\mu \mathrm{L}$ Hyb \#2, $4 \mu \mathrm{L} \mathrm{Hyb} \mathrm{\# 3,} \mathrm{and} 4 \mu \mathrm{L}$ Hyb \#4. The Capture Baits Master Mix was prepared by mixing $2.5 \mu \mathrm{L}$ capture probe with $0.5 \mu \mathrm{L}$ RNase Block. The Library Master Mix was incubated at $95^{\circ} \mathrm{C}$ for 5 min with a subsequent incubation at $65^{\circ} \mathrm{C}$. The Hybridization Master Mix was incubated at $65^{\circ} \mathrm{C}$ for $2 \mathrm{~min}$. The Capture Baits Master Mix was incubated at $65^{\circ} \mathrm{C}$ for 5 min. On a $65^{\circ} \mathrm{C}$ thermal cycler, 3.5 $\mu \mathrm{L}$ Library Master Mix and 6.5 $\mu \mathrm{L}$ Hybridization Master Mix were transferred to the Capture Baits Master Mix. The hybridization was performed for $22 \mathrm{~h}$ at $65^{\circ} \mathrm{C}$ in a thermal cycler. DNA-RNA hybrids were selected with Dynabeads MyOne Streptavidin C1 (Thermo Fisher, Cambridge, UK) according to the MYbaits manufacturer's instructions and were finally resuspended in $50 \mu \mathrm{l} 1 \times$ TE buffer.

Targeted capture amplification

The captured DNA fragments were amplified with the following reaction set-up: $1 \mu \mathrm{L}, 5 \mu \mathrm{L}$, or $10 \mu \mathrm{L}$ MyOne Streptavidin C1 beads; $0.3 \mu \mathrm{M}$ Illumina Primer Mix; and $25 \mu \mathrm{L} 2 \times$ Kapa HiFi HotStart Ready Mix in a total volume of $50 \mu \mathrm{L}$. We used the following PCR program: $94^{\circ} \mathrm{C} 3 \mathrm{~min}, 25 \times\left[94^{\circ} \mathrm{C} 30 \mathrm{~s}, 60^{\circ} \mathrm{C} 30\right.$ $\left.\mathrm{s}, 68^{\circ} \mathrm{C} 4 \mathrm{~min}\right], 68^{\circ} \mathrm{C} 10 \mathrm{~min}$. The ramp rate was set to $3.0^{\circ} \mathrm{C} / \mathrm{s}$. We performed three individual amplification reactions for each library to minimize PCR biases and PCR duplications and to study the effect of varying template amounts on the PCR yield of the different insert-size libraries. The obtained amounts were ffor $1 \mu \mathrm{L}, 5 \mu \mathrm{L}$, or $10 \mu \mathrm{L}$ MyOne Streptavidin C1 beads respectively): $4.8 \mu \mathrm{g}, 3.1 \mu \mathrm{g}$, $2.3 \mu \mathrm{g}$ for the 4-kb insert library; $3.8 \mu \mathrm{g}$, $4.5 \mu \mathrm{g}, 3.6 \mu \mathrm{g}$ for the 5-kb insert library, and $2.5 \mu \mathrm{g}, 4.3 \mu \mathrm{g}, 4.7 \mu \mathrm{g}$ for the $7-\mathrm{kb}$ insert library.

\section{Size selection of the}

\section{enriched R-gene library}

Sage ELF size selection was performed as previously described, but the separation time was adjusted to the fragment size of interest (4-kb amplicons: 1 h 6 min; 5-kb and 7-kb amplicons: 2 h 18 min), followed by a $0.4 \times$ AMPure XP bead purification.

\section{PacBio library assembly}

PacBio libraries were constructed according to the Pacific Biosciences 5-kb Template Preparation and Sequencing protocol, version 6 (www.pacificbiosciences.com/support/pubmap/documentation.html). Samples were prepared using the DNA Template Prep Kit 3.0 (Pacific Biosciences, Menlo Park, CA). To avoid loss of material, only the first bead purification step prior to the DNA damage repair step was conducted using a $0.45 x$ ratio of AMPure PB magnetic beads (Pacific Biosciences). All other remaining clean-up steps were performed using a $0.6 \times$ ratio of $A M P$ ure $\mathrm{PB}$ beads. Finally, the libraries were purified with 2 consecutive 0.6x AMPure PB bead clean-up reactions and eluted in $10 \mu \mathrm{L}$ PacBio EB buffer.

\section{PacBio loading and sequencing}

The SMRTcell loading protocol was generated with the PacBio Binding Calcu- lator v.2.3.1.1. To maximize SMRTcell yield, the concentration on the plate was set to $0.1 \mathrm{nM}$ instead of using the default value of $0.01 \mathrm{nM}$. The sequencing primer was annealed by heating the sample to $80^{\circ} \mathrm{C}$ followed by a slow cool-down of $0.1^{\circ} \mathrm{C} / \mathrm{s}$ to $25^{\circ} \mathrm{C}$. For each library, 2 SMRTcells were sequenced with the PacBio P6-C4 chemistry and 240-min movies.

\section{Evaluation of reads and subreads}

Reads of insert (Rol) were generated using PacBio's SMRTpipe ReadsOflnsert algorithm. Filtered subreads (SR) were retrieved from PacBio's SMRTportal web interface. Illumina adapter sequences $64 \mathrm{bp}$ in length were removed from the $5^{\prime}$ and 3 ' ends of the Rol and SR using cutadapt-1.8.1 (6) (Supplementary Material). SR and Rol statistics were calculated with abyss-1.9.0 (7) (Supplementary Material), and modal values were calculated with a custom Python script (see github repository: https://github. com/mgiolai/Ren-seq). The Rol were aligned to the S. verrucosum VER54 draft genome with the "bwa-mem -x pacbio" setting (https://arxiv.org/abs/1303.3997). To assess the sequencing coverage of the assembled contigs, we aligned the Rol to each corresponding assembly with the "bwa-mem -x pacbio" setting. To evaluate how many Rol and SR contain at least one bait sequence, the SR and Rol sequences of each library were searched separately against the bait sequences using BLAST-2.2.28+ with the following arguments: "-num_threads 8 -outfmt 6" (8) (Supplementary Material). The BLASTN results were analyzed with a custom Python script that only scores $80 \%$ sequence identity between read

Table 1. Size and quantity of recovered DNA for the size-selection run of the amplified TruSeq library on the SageELF.
\begin{tabular}{|c|c|c|c|}
\hline \\
Fraction & Size SageELF (kb) & \multicolumn{1}{c|}{ Size BioAnalyzer (kb) } & DNA recovered ( $\boldsymbol{\mu g}$ ) \\
\hline 1 & 10.7 & Not assessed & 0.01 \\
\hline 2 & 7.7 & 7.3 & 0.59 \\
\hline 3 & 5.7 & 5.5 & 1.73 \\
\hline 4 & 4.2 & 4.2 & 1.28 \\
\hline 5 & 3.1 & 3.1 & 0.51 \\
\hline 6 & 2.3 & 2.4 & 0.18 \\
\hline 7 & 1.7 & 1.7 & 0.04 \\
\hline 8 & 1.4 & Not assessed & 0.02 \\
\hline 9 & 0.8 & Not assessed & 0.01 \\
\hline 10 & 0.5 & Not assessed & 0.02 \\
\hline 11 & 0.3 & Not assessed & Not measurable \\
\hline 12 & 0.1 & Not assessed & Not measurable \\
\hline
\end{tabular}


and bait sequences over at least $96 \mathrm{bp}$ as a hit. R-gene detection was performed with the NLR-parser-v1.0 (9) using default values.

\section{NB-LRR gene assembly}

The raw PacBio sequence data sets are stored in .bax.h5 files. A custom pipeline was built in SMRTanalysis 2.3.0 to assemble the data. The main changes to the standard SMRTanalysis assembly pipeline were filtering the raw reads from Illumina adapters and whitelisting (filtering out reads where adapter sequences were not removed).

The Illumina adapters, which are ligated to the DNA during library preparation, were removed before assembling the sequences. We used a custom script (available at https://github.com/ paajanen/RenSeq) to trim the adapters off with the h5-raw files as input. This custom script trims each read by $64 \mathrm{bp}$ (the length of the adapter).

We used BLASR (10) to align the adapter sequences to the edited files with a whitelisting script to filter out reads where the adapter was not removed. Those reads were $1 \%-2 \%$ of the raw reads, and they represent chimeric reads (either from physical ligation of two amplicons or virtual chimeras with PacBio software not recognizing the SMRTbell adapters).

The edited bax.h5 files were fed into the SMRTanalysis HGAP3 protocol (11) along with a parameter file that contained the whitelisting. All of the scripts are available at github (https://github.com/ paajanen/RenSeq).

\section{Results and discussion}

High molecular weight S. verrucosum VER54 genomic DNA was extracted (Supplementary Figure S1) and sheared to $8 \mathrm{~kb}$ using the Megaruptor (Supplementary Figure S2). Due to the tight size distribution, no additional size-selection step (with its associated loss of material) was necessary before long-insert Illumina TruSeq library construction.

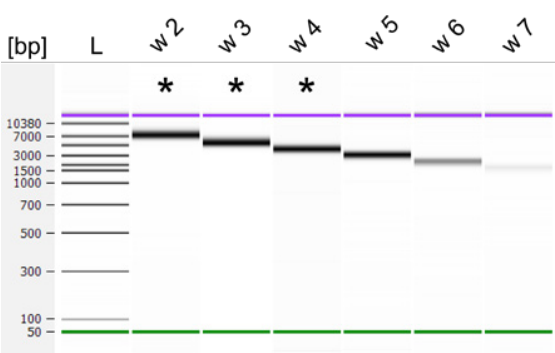

Figure 2: SageELF size selection of the PreCRrepaired TruSeq library PCR. The PreCR-repaired TruSeq library was size-selected on the SageELF and the selection profile was assessed on the Agilent Bioanalyzer. To test the performance of long insert size libraries in the MYbaits capture protocol, the 7-kb (Well 2), 5-kb (Well 3) and 4-kb size fractionated DNA (Well 4) were used for individual captures - these wells are marked with an asterisk.

To assess the possible benefits of PreCR DNA repair on library construction, we compared the yield and size distribution of two 8-kb libraries with and without DNA repair. Equal amounts of both TruSeq libraries were amplified using the Kapa HiFi polymerase. In both cases, the PCR yield was sufficient for size selection on the SageELF (5 $\mu \mathrm{g}$ maximum input per gel cassette). We observed a substantial shift in fragment size distribution in the repaired and non-repaired sample after PCR. The PreCR-repaired library showed a decrease from $7.4 \mathrm{~kb}$ to $5.3 \mathrm{~kb}$, whereas the non-DNA repaired sample decreased from $7.0 \mathrm{~kb}$ to $5.0 \mathrm{~kb}$ (Supplementary Figure S2). From this, we concluded that the amplification profile of the Illumina library was little affected by PreCR repair. Lower quality DNA or DNA sheared by other methods (e.g., sonication) might benefit from this step. However, as PreCR repair could be beneficial to the quality of longer DNA molecules, we decided to proceed with the PreCR-treated library.

To minimize the preferential amplification of smaller DNA molecules by PCR, we tested different PCR conditions and commercially available long-range PCR mixes such as the KAPA Long Range PCR Kit and the QIAGEN Long Range PCR Kit (Supplementary Material). Both are advertised to amplify DNA fragments longer than $10 \mathrm{~kb}$. From our results, we concluded that amplification of the TruSeq library with the long-range PCR mixes or various PCR conditions did not lead to better results (e.g., longer PCR products). We preferred using Kapa HiFi for subsequent reactions as this enzyme has minimal GC bias and high proofreading activity (12).

The SageELF is designed to fractionate DNA into 12 separate elution wells with defined fragment sizes. SageELF size selection of a $5-\mu \mathrm{g}$ TruSeq library yielded $\sim 4.3 \mu \mathrm{g}$ of DNA (87\% recovery). This shows that a single size-selection run using $5 \mu \mathrm{g}$ of input DNA on the Sage ELF produces enough DNA for a capture approach. This amount can be achieved with one or two Kapa HiFi PCR reactions.

We selected 3 SageELF size fractions (4-kb, 5-kb, and 7-kb) for bait hybridization (Table 1, Figure 2). The DNA/ RNA bait hybridization to capture resistance gene sequences was performed individually on the 4-kb, 5-kb, and 7-kb fractions according to the MYbaits target enrichment protocol but with minor modifications (see "Materials and methods" section).

Despite all optimization steps, we still observed a small decrease in library size after the amplification of the MYbaits capture reactions. The 4-kb library was reduced by $0.2 \mathrm{~kb}$, the $5-\mathrm{kb}$ library by $0.7 \mathrm{~kb}$, and the 7-kb library by $1.5 \mathrm{~kb}$ (Supplementary Figure S3). The yield of 2 PCR reactions after 25 cycles of amplification was $>5 \mu \mathrm{g}$.

We loaded $5 \mu \mathrm{g}$ of each library on the SageELF (Supplementary Tables S1-S3) for a final size selection. The yield for the 4-kb capture approach was $\sim 1.4 \mu \mathrm{g}$ DNA with a size $>3.9 \mathrm{~kb}$; for the 5-kb capture approach it was $\sim 1.2 \mu \mathrm{g}$ DNA with a size $>5.1 \mathrm{~kb}$; and for the $7 \mathrm{~kb}$ capture approach it was $\sim 1.7$ $\mu \mathrm{g}$ DNA with a size $>6.2 \mathrm{~kb}$ (Figure 3). Each fraction was used to construct a PacBio library according to the PacBio 5-kb Template Preparation protocol. This resulted in PacBio libraries with modal sizes of $4.6 \mathrm{~kb}, 5.7 \mathrm{~kb}$, and $7.0 \mathrm{~kb}$ (further

Table 2. Overview of the sequencing performance values.

\begin{tabular}{|c|c|c|c|c|c|c|c|c|c|c|}
\hline $\begin{array}{l}\text { Library insert } \\
\text { size }(\mathbf{k b})\end{array}$ & $\begin{array}{l}\text { Movie length } \\
\text { (min) }\end{array}$ & $\begin{array}{c}\text { PR: Mean } \\
\text { length post-filter (kb) }\end{array}$ & $\begin{array}{l}\text { PR: } \mathbf{N}_{50} \text { length } \\
\text { post- filter }(\mathbf{k b})\end{array}$ & $\begin{array}{l}\text { PR: Mean quality } \\
\text { post-filter }\end{array}$ & $\begin{array}{l}\text { Rol: Mean } \\
\text { length (kb) }\end{array}$ & $\begin{array}{l}\text { Rol: Mode } \\
\text { length (kb) }\end{array}$ & $\begin{array}{l}\text { Rol: } \mathbf{N}_{50} \\
\text { length (kb) }\end{array}$ & $\begin{array}{l}\text { Rol: Mean } \\
\text { quality (\%) }\end{array}$ & $\begin{array}{c}\text { Rol: Total } \\
\text { length (Mb) }\end{array}$ & $\begin{array}{c}\text { Mean } \\
\text { passes }\end{array}$ \\
\hline 4 & 240 & 17.0 & 25.0 & 0.84 & 3.7 & 3.7 & 3.8 & 97.74 & 109.9 & 6.0 \\
\hline 5 & 240 & 15.4 & 24.2 & 0.84 & 4.2 & 4.8 & 4.8 & 97.22 & 68.6 & 5.3 \\
\hline 7 & 240 & 14.7 & 25.6 & 0.85 & 4.8 & 6.5 & 6.2 & 96.90 & 42.4 & 4.7 \\
\hline
\end{tabular}



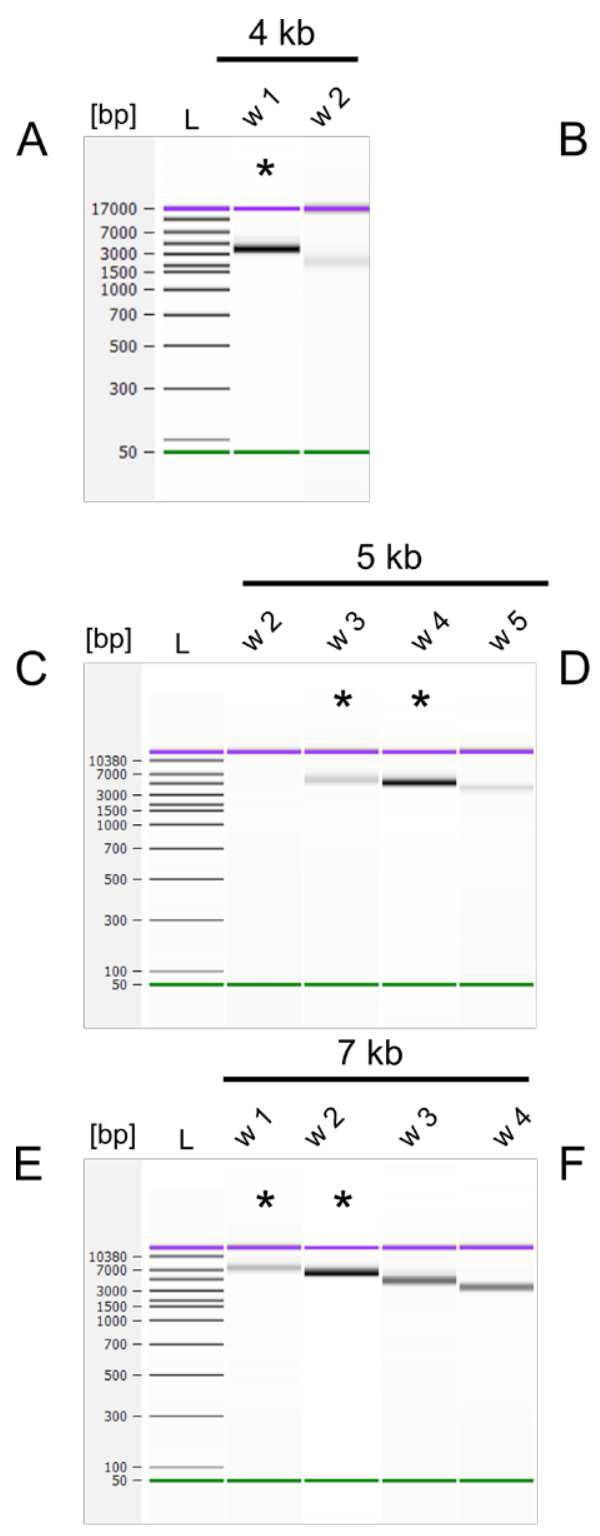
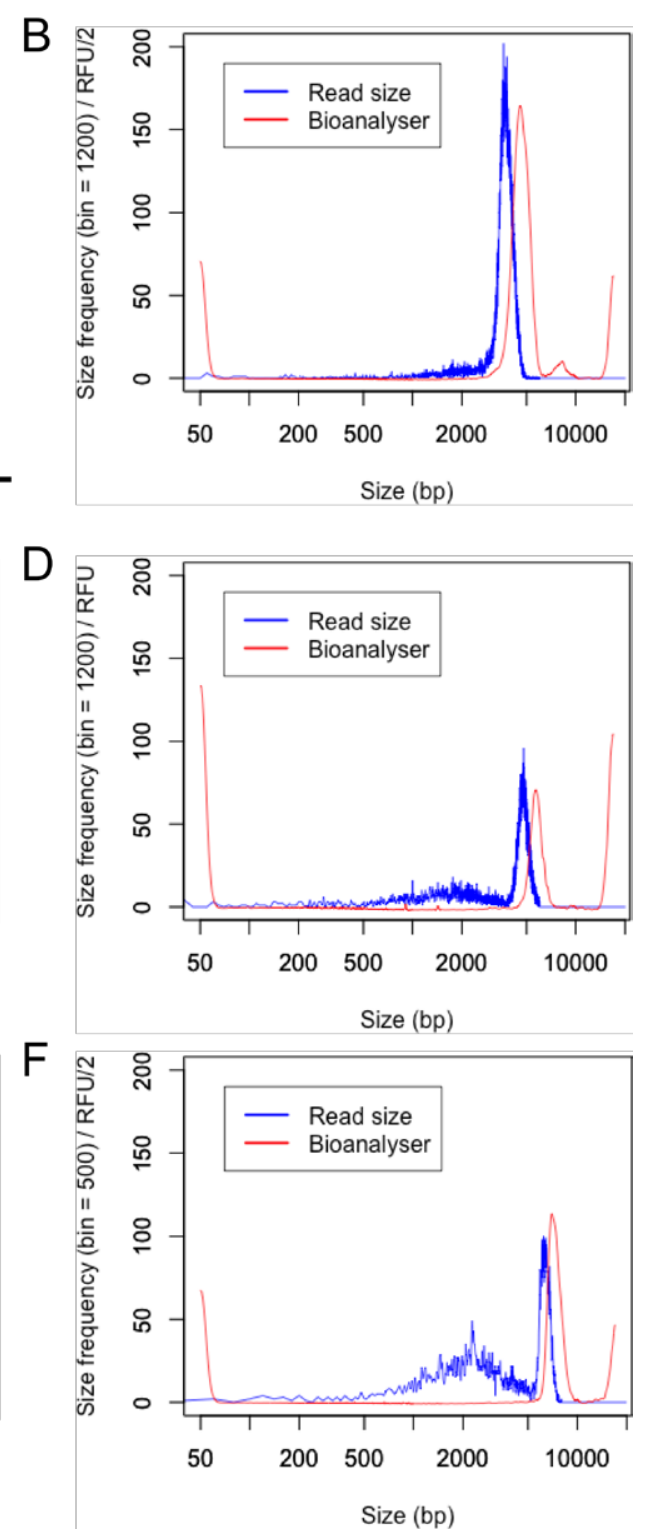

Figure 3. SageELF size selection of the captured library amplifications and overlay of the trimmed reads of insert (Rol) with the Bioanalyzer electropherograms. ( $A, C, E)$ SageELF size selection of the capture amplification for the 4-kb, 5-kb, and 7-kb libraries. For the 4-kb library, Well 1 (3.9 kb) was used; for the 5-kb library, Wells $3(5.6 \mathrm{~kb})$ and 4 (5.1 kb) were used; and for the 7-kb library, Wells $1(7.6 \mathrm{~kb})$ and $2(6.2 \mathrm{~kb})$ were used. (Used wells are marked with an asterisk). (B, D,E) The Bioanalyzer electropherograms of the final 4-kb, 5-kb, and 7-kb PacBio libraries are highlighted in red. The blue trace shows the Illumina adaptercurated Rol distribution fitted to the library size. The Rol distribution is close to the target library size. Whereas in the 4-kb sequencing run only a small number of short fragments were sequenced, the number of fragments sequenced increased for the 5-kb and 7-kb libraries.

Table 3. Subread (SR) statistical comparison between the three R-gene enrichment sequencing (RenSeq) libraries and the PacBio-LITS 6-kb NA12878 library.

\begin{tabular}{|c|c|c|c|c|c|c|}
\hline $\begin{array}{l}\text { Library insert } \\
\text { size (kb) }\end{array}$ & $\begin{array}{l}\text { SR: Number } \\
\text { of reads }\end{array}$ & $\begin{array}{l}\text { SR: Min read } \\
\text { length (kb) }\end{array}$ & $\begin{array}{l}\text { SR: Max read } \\
\text { length (kb) }\end{array}$ & $\begin{array}{l}\text { SR: Mean read } \\
\text { length (kb) }\end{array}$ & $\begin{array}{l}\text { SR: Mean } N_{50} \\
\text { read length (kb) }\end{array}$ & $\begin{array}{c}\text { Total SR } \\
\text { (bp) }\end{array}$ \\
\hline 4 & 564,678 & 0.5 & 41.0 & 3.7 & 4.0 & $2.1 \times 10^{9}$ \\
\hline 5 & 465,084 & 0.5 & 39.3 & 4.0 & 5.0 & $1.9 \times 10^{9}$ \\
\hline 7 & 441,819 & 0.5 & 43.9 & 4.9 & 6.6 & $2.2 \times 10^{9}$ \\
\hline 6 (PacBio-LITS) & 856,571 & 0.5 & 33.9 & 3.0 & 4.5 & $2.5 \times 10^{9}$ \\
\hline
\end{tabular}

referred as $4 \mathrm{~kb}, 5 \mathrm{~kb}$, and $7 \mathrm{~kb}$ ) (Supplementary Figure S4). We sequenced each library on 2 SMRT cells using the latest P4-C6 chemistry and 240-min movies. The mean polymerase read length for all libraries was $>14 \mathrm{~kb}$, with the highest value of $17 \mathrm{~kb}$ for the $4 \mathrm{~kb}$ library (Table 2 and Supplementary Figure S5). The $\mathrm{N}_{50}$ value for the polymerase read length was $>24 \mathrm{~kb}$ for all libraries, with reads as long as $40 \mathrm{~kb}$. In the 4-kb library we observe fewer small Rol compared with the $5-\mathrm{kb}$ and $7-\mathrm{kb}$ libraries, indicating that even when using SageELF size selection there are still small molecules present that are preferentially sequenced on the PacBio RSIl sequencing platform (Figure 3). This leads to a mean Rol value that is lower than the library size, particularly in the 7-kb library. The $\mathrm{N}_{50}$ values and graphical read length plots, however, show a clear enrichment for the desired insert size (Table 2, Figure 3, and Supplementary Figure S5). A mean pass value of 8 was achieved for all libraries. This is due to persistent short library molecules (Figure 3) that contribute to the increased mean pass values. We calculated the pass value for the fragments that correspond to the targeted size: the mean pass value was 6 for the 4-kb library (reads of 3-5 $\mathrm{kb}), 5.3$ for the 5-kb library (reads of 4-6 $\mathrm{kb}$ ), and 4.7 for the 7-kb library (reads of 5.5-8 kb). The mean polymerase read length for all libraries was $>14 \mathrm{~kb}$, with the highest value of $17 \mathrm{~kb}$ for the 4-kb library (Table 2 and Supplementary Figure S5). The $\mathrm{N}_{50}$ values for the polymerase read length were $>24 \mathrm{~kb}$ for all libraries, with reads as long as $40 \mathrm{~kb}$ indicating that long inserts can be sequenced effectively with the PacBio P6-C4 chemistry.

In comparison to other capturebased sequencing protocols, such as the PacBio-LITS protocol (13), we were able to generate a higher fraction of reads with the targeted fragment size (Supplementary Figure S6). A 6-kb HapMap NA12878 PacBio-LITS capture approach (2 SMRT cells) generated a visible enrichment for 6 -kb inserts but with a high number of reads $<6 \mathrm{~kb}$ (Table 3). While the NA12878 approach used the older PacBio P5-C3 chemistry with somewhat shorter mean read lengths, (PacBio reports read lengths of $8.5 \mathrm{~kb}$ for P5-C3 and >10 kb for P6-C4 chemistries) the PacBio-LITS study obtained a mean read length of $8.0 \mathrm{~kb}$ for the $6-\mathrm{kb}$ 
A

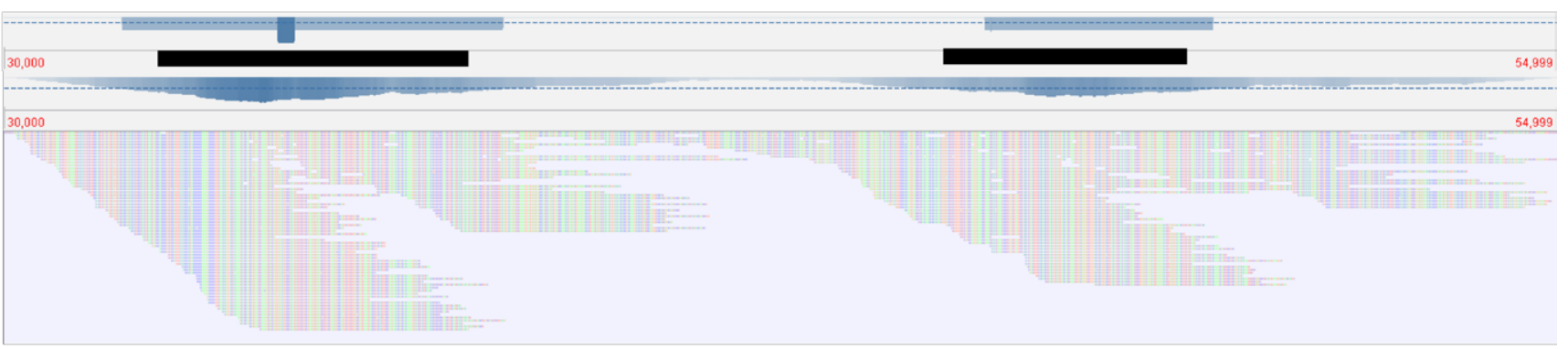

B

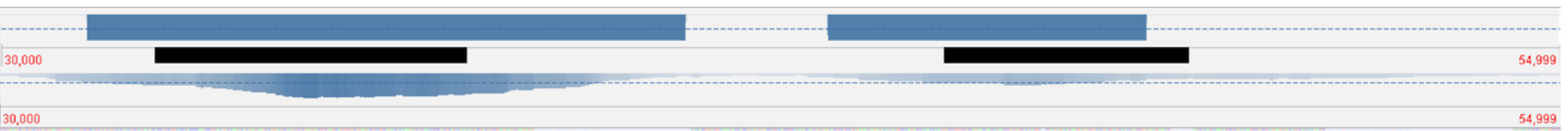

C

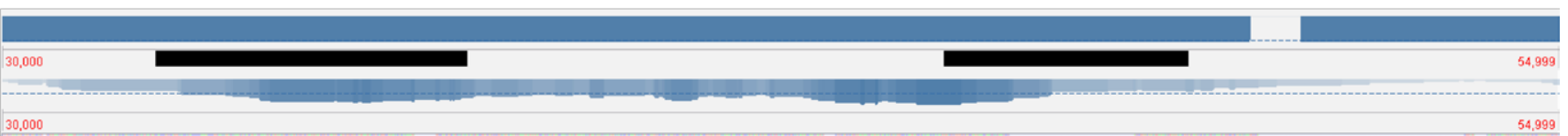

Figure 4. Reads of insert (Rol) and assembled contigs were mapped to a scaffold of a draft Solanum verrucosum VER54 chromosome 10 assembly. Shown here are the Rol and HGAP assembled contigs (blue bars) of (A) a 4-kb library, (B) a 5-kb library, and (C) a 7-kb library mapped to a 25-kb region of Solanum verrucosum VER54 chromosome 10 that contains 2 R-gene sequences (black bars). The image shows a clear enrichment of reads in R-gene coding regions. The larger the insert size, the more efficiently the intergenic region between two R-genes can be bridged. The coverage of the intergenic region between the two R-genes is lower with reads of the 4-kb library than with reads obtained from the 7-kb library. This influences the contig size; whereas for the 4-kb and 5-kb libraries each R-gene is assembled in a separate contig, the $7 \mathrm{~kb}$ contig contains both R-genes and the sequence between the 2 R-gene coding regions.

HapMap NA12878 capture approach and read lengths $>33 \mathrm{~kb}$. This suggests that the P5-C3 chemistry is able to sequence $6-\mathrm{kb}$ insert libraries efficiently and that many of the short reads for the NA12878 in the PacBio-LITS study are probably due to the lack of a second size selection after post-capture PCR, which we found to be important for maintaining the correct library sizes.

To compare the capture efficiency between the three library sizes, we determined the fraction of on-target reads that contain sequences nearly identical to the probes in the bait library. To do this, SR or Rol were searched by BLAST against a database of the bait sequences. The criterion for a positive score was that an SR or Rol sequence must possess at least 1 region with $80 \%$ sequence identity over 96 bp to the bait library, as described by Jupe et al. (3). For the $7-\mathrm{kb}$ library this resulted in $44 \%$ of Rol containing $\geq 1$ bait sequence according to the set threshold and values of $56 \%$ and $55 \%$ for the $4-\mathrm{kb}$ and 5 -kb libraries, respectively. For the SR, the numbers are lower. Between $30.5 \%$ and $35 \%$ of the SR contain $\geq 1$ bait sequence (Table 4). This can be explained by the lower sequence accuracy of the SR (86.4\% for $4 \mathrm{~kb}$ and $5 \mathrm{~kb}, 86.7 \%$ for $7 \mathrm{~kb})$ in comparison to the Rol $(97.74 \%$ for $4 \mathrm{~kb}, 97.22 \%$ for $5 \mathrm{~kb}$, and $96.90 \%$ for $7 \mathrm{~kb}$ ).

We used the NLR-parser software (9) to determine the number of R-gene motif-containing sequences in the data generated from each library. In the 7-kb insert library, $27.7 \%$ of all reads were annotated as R-gene motif-containing sequences. For the $4-\mathrm{kb}$ and $5-\mathrm{kb}$ libraries, this number is higher in relation to the total number of reads (for both above 34.9\%). The 7-kb library showed the highest percentage (24\%) of complete annotated hits (full R-gene contained within a Rol) in relation to the total number of NLR-parser hits. The 5-kb library contained $21.6 \%$ reads with complete annotated hits, with $16.8 \%$ complete hits for the 4-kb library (Table 4).

For the assembly, we trimmed the reads to remove the Illumina adapters and excluded chimeric sequences from the data set (see "Materials and methods" section). Read clustering approaches are beneficial for assem-

Table 4. Number and percentage of subreads (SR) and reads of insert (Rol) that contain at least 1 bait hit with $\mathbf{8 0 \%}$ sequence identity over 96 bp.

\begin{tabular}{|c|c|c|c|c|c|c|c|c|c|c|}
\hline $\begin{array}{c}\text { Library } \\
\text { insert } \\
\text { size (kb) }\end{array}$ & $\begin{array}{c}\text { SR: } \\
\text { Number } \\
\text { of reads }\end{array}$ & $\begin{array}{c}\text { SR: Number } \\
\text { of reads } \\
\text { with baits }\end{array}$ & $\begin{array}{c}\text { \% SR } \\
\text { containing } \\
\text { baits }\end{array}$ & $\begin{array}{c}\text { Number of } \\
\text { reads }\end{array}$ & $\begin{array}{c}\text { Rol: Number } \\
\text { of reads with } \\
\text { baits }\end{array}$ & $\begin{array}{c}\text { Rol: NLR- } \\
\text { parser } \\
\text { hits }\end{array}$ & $\begin{array}{c}\text { Rol: NLR-parser } \\
\text { hits scored as } \\
\text { complete }\end{array}$ & $\begin{array}{c}\% \text { Rol } \\
\text { with } \\
\text { baits }\end{array}$ & $\begin{array}{c}\% \text { Rol with } \\
\text { NLR-parser } \\
\text { hits }\end{array}$ & $\begin{array}{c}\% \text { Rol NLR-parser } \\
\text { hits scored as } \\
\text { complete }\end{array}$ \\
\hline 4 & 564,678 & 193,655 & 34.4 & 29,773 & 16,761 & 10,393 & 1751 & 56.3 & 34.9 & \\
\hline 5 & 465,084 & 162,657 & 35.0 & 16,372 & 9092 & 5818 & 1258 & 55.5 & 35.5 & \\
\hline 7 & 441,819 & 134,572 & 30.5 & 8770 & 3855 & 2430 & 583 & 44.0 & 21.6 & 27.7 \\
\hline
\end{tabular}


Table 5. Assembly statistics for the 4-kb, 5-kb, and 7-kb libraries.

\begin{tabular}{|c|c|c|c|c|c|c|c|c|}
\hline $\begin{array}{l}\text { Library insert } \\
\text { size (kb) }\end{array}$ & $\begin{array}{l}\text { Number of } \\
\text { contigs }\end{array}$ & $\begin{array}{l}\text { Minimal contig } \\
\text { length (bp) }\end{array}$ & Contig $\mathrm{N}_{80}$ & Contig $\mathrm{N}_{50}$ & Contig $\mathrm{N}_{20}$ & Contig E-size & $\begin{array}{l}\text { Maximal contig } \\
\text { length (bp) }\end{array}$ & $\begin{array}{c}\text { Sum of base } \\
\text { pairs assembled }\end{array}$ \\
\hline 4 & 1237 & 516 & 4208 & 8542 & 14,018 & 12,243 & 136,022 & $6,998,134$ \\
\hline 5 & 1171 & 510 & 5391 & 10,571 & 18,195 & 14,591 & 139,788 & $8,269,857$ \\
\hline 7 & 1126 & 503 & 7530 & 14,350 & 25,387 & 19,615 & 143,859 & $9,959,491$ \\
\hline
\end{tabular}

bling highly similar sequences with PacBio data (11,14-16). To integrate this with our assembly pipeline, we used the PacBio SMRTanalysis HGAP3 algorithm (11) with empirically determined custom parameters (see github repository: https://github. com/paajanen/RenSeq).

To study the assembly performance of the 3 libraries, we analyzed the coverage of the 4-kb, 5-kb, and 7-kb HGAP contigs. As the bait library is specifically designed for R-gene coding sequences (17), coding and non-coding regions are unequally covered (Figure 4); therefore, we counted the number of reads mapping to each contig. Contigs of the 4-kb library are supported by an average of 31 Rol, contigs of the $5-\mathrm{kb}$ library by an average of $17 \mathrm{Rol}$, and contigs of the 7-kb library by an average of $11 \mathrm{Rol}$.

Whereas for the 4-kb library a total of 1237 contigs with an $\mathrm{N}_{50}$ value of 8.5-kb was generated, we observed a better assembly performance for the 5-kb (1171 contigs, $N_{50}=10.6$ $\mathrm{kb}$ ) and 7-kb (1126 contigs, $\mathrm{N}_{50}=14.3 \mathrm{~kb}$ ) libraries (Table 5). We used the NLR-parser to count the number of R-gene motif-containing contigs. The NLR-parser analysis resulted in 485, 468, and 402 R-gene motif-containing contigs for the 4-kb, 5-kb, and 7-kb assemblies, respectively. The number of complete annotated contigs increases with the library insert size from $48.4 \%(4 \mathrm{~kb})$ to $53.2 \%(5 \mathrm{~kb})$ and $57.0 \%(7 \mathrm{~kb})$. To determine the number of R-genes in the assemblies, we annotated the NLR-parser result files manually by considering that long contigs can harbor more than one R-gene (Figure 4) but are listed as a single hit by the software. We sought to compare these numbers with the R-genes detected in the S. verrucosum VER54 genome assembly. For this, we performed an NLR-parser analysis on the scaffolds of the reference genome and annotated the number of R-genes in the genome manually based on the motif arrangement, as previously described $(4,9)$. In the draft S. verrucosum VER54 assembly, we discovered 409 R-genes in comparison to 502 R-genes, 488 R-genes, and 429 R-genes in the 4-kb, 5-kb, and 7-kb assemblies, respectively. These numbers are similar to the numbers of annotated R-genes for the potato and tomato species: Solanum tuberosum clone DM (704 R-genes) (3), Solanum americanum accession 954750186 (649 R-genes) (17), and Solanum pimponellifolium LA1589 (355 R-genes ) (5).

R-genes can occur as isolated genes or as clusters of varying size $(3,4,18)$. Besides the coding sequence itself, the non-coding and intergenic regions between two R-genes in a cluster can contain regulatory sequences such as promoter elements (19). Therefore, large insert libraries might have an advantage in linking the potentially polymorphic intergenic regions with the more conserved coding sequence. To analyze the benefits of long-insert libraries, we looked at the coverage of intergenic regions within R-gene clusters in our draft S. verrucosum VER54 genome assembly (Paajanen et al., data not shown), which was generated using Illumina 250-bp paired-end (DISCOVAR de novo assembly) and Illumina Long Mate Pair sequencing (20), and Dovetail Hi-C scaffolding (21). The Rol and the HGAP contigs of the three PacBio libraries were mapped separately to an S. verrucosum VER54 chromosome 10 scaffold containing two predicted $\mathrm{R}$-gene coding regions to determine if longer insert sizes can capture the region between R-genes where promoters and terminator sequences could reside. The alignments clearly indicate an increasing coverage of the $25-\mathrm{kb}$ region with an increasing library insert size. Whereas the 4-kb library Rol annotate the 2 R-genes, the 7-kb library Rol close the genomic gap between 2 adjacent R-genes. As a result, the reads of the 7-kb library are assembled to a single continuous contig containing the intergenic region and both $\mathrm{R}$-genes, whereas the 4-kb reads are assembled to 3 different contigs without the intergenic sequence information (Figure 4).

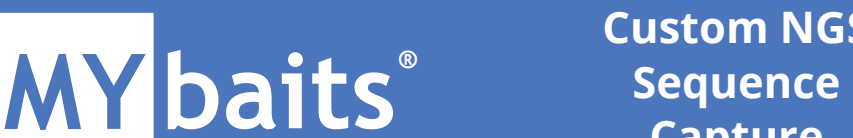 Capture}

\section{The trusted solution for targeted next-gen sequencing of any organism}

\section{Rapidly sequence thousands of} loci of interest with efficient and affordable in-solution enrichment

Target DNA sequences from animals, plants, and microbes, even from degraded specimens

Complete, user-friendly kits with free bait \& project design assistance Phylogenetics, variant discovery, \& more

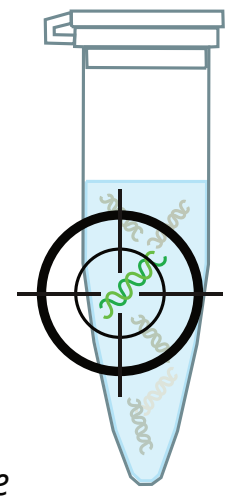

Begin your project at mycroarray.com/mybaits

MYcroarray

THE OLIGO LIBRARY COMPANY Scientist-owned in Ann Arbor, MI, USA
Talk with an

NGS expert today! info@mycroarray.com +1 (734) 998-0751 
By optimizing the shearing, size selection, and PCR amplification steps of the RenSeq capture protocol, our library construction strategy can be used for targeted sequencing of inserts up to 7 -kb long with high quality (>96\% sequence accuracy). Although the Rol yield per SMRTcell decreases with increasing insert length, long-insert libraries lead to a higher sequence coverage upstream and downstream of the targeted sequence. This makes the optimized RenSeq protocol not only interesting for very accurate long-read $\mathrm{R}$-gene enrichment but also as a robust and reproducible technique for the hybridization-based specific capture of fragments up to 7-kb in any genomic context-and it could be used for gap filling, other types of genome finishing, or structural variation verification.

\section{Author contributions}

M.G. conducted and validated the experiments. P.P. established the assembly pipeline and analyzed the data. W.V. supervised and provided expertise for the experiments. K.W. and F.J. designed the bait libraries for the experiment. K.W. and J.D.G. provided the bait libraries used in the experiment. I.H. provided three additional bait libraries. G.B. provided the S. verrucosum VER54 material. L.P.A. contributed a custom script to create Figure 3. D.B. performed the PacBio RSII run. M.G. wrote the manuscript. W.V. and M.D.C. edited the manuscript. M.D.C. designed and supervised the study.

TThe HapMap NA12878 data set was kindly provided by Min Wang, Adam C. English and Richard A. Gibbs. We thank Darren Heavens for assistance with size selection. This work was strategically

\section{Acknowledgments}

funded by the BBSRC, Institute Strategic Programme Grant BB/J004669/1 at the Earlham Institute (formerly The Genome Analysis Centre, Norwich), and BBSRC Grant "Controlling important diseases in potato by cloning functional NB-LRR-type resistance genes" BB/L009757/1). Nextgeneration sequencing was delivered via the BBSRC National Capability in Genomics (BB/J010375/1) at Earlham Institute, by members of the Platforms and Pipelines Group.

\section{Competing interests}

M.D.C. owns shares in Pacific Biosciences.

\section{References}

1. Jones, J.D.G. and J.L. Dangl. 2006. The plant immune system. Nature 444:323-329.

2. Jupe, F., X. Chen, W. Verweij, K. Witek, J.D.G. Jones, and I. Hein. 2014. Genomic DNA Library Preparation for Resistance Gene Enrichment and Sequencing (RenSeq) in Plants. In Plant-Pathogen Interactions, P. Birch, J.T. Jones, and J.I.B. Bos, eds. (Totowa, NJ: Humana Press), pp. 291-303.

3. Jupe, F., K. Witek, W. Verweij, J. Sliwka, L. Pritchard, G.J. Etherington, D. Maclean, P.J. Cock, et al. 2013. Resistance gene enrichment sequencing (RenSeq) enables reannotation of the NB-LRR gene family from sequenced plant genomes and rapid mapping of resistance loci in segregating populations. Plant J. 76:530-544.

4. Jupe, F., L. Pritchard, G.J. Etherington, K. MacKenzie, P.J. Cock, F. Wright, S.K. Sharma, D. Bolser, et al. 2012. Identification and localisation of the NB-LRR gene family within the potato genome. BMC Genomics 13:75.

5. Andolfo, G., F. Jupe, K. Witek, G.J. Etherington, M.R. Ercolano, and J.D.G. Jones. 2014. Defining the full tomato NB-LRR resistance gene repertoire using genomic and cDNA RenSeq. BMC Plant Biol. 14:120.

6. Martin, M. 2011. Cutadapt removes adapter sequences from high-throughput sequencing reads. EMBnet J. 17:10-12.

7. Simpson, J.T., K. Wong, S.D. Jackman, J.E. Schein, S.J.M. Jones, and I. Birol. 2009. ABySS: A parallel assembler for short read sequence data. Genome Res. 19:1117-1123.

8. Altschul, S.F., W. Gish, W. Miller, E.W. Myers, and D.J. Lipman. 1990. Basic local alignment search tool. J. Mol. Biol. 215:403-410.
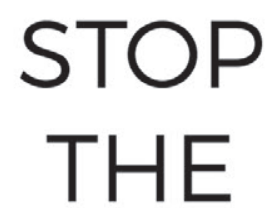

Available through distributors
DRIP

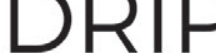

For more information go to ww
The Better Way to Pipette GTWobble-not $\square^{m}$ No Drip Design

2-tiers $/ 2$ steps $=2 \mathrm{X}$ contact in your controller $=$ better seal

For Hassle-Free Serological Pipetting

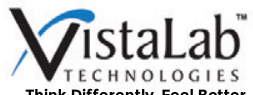
Low Insertion Force Serological Pipet
9. Steuernagel, B., F. Jupe, K. Witek, J.D.G. Jones, and B.B.H. Wulff. 2015. NLR-parser: rapid annotation of plant NLR complements. Bioinformatics 31:1665-1667.

10. Chaisson, M.J. and G. Tesler. 2012. Mapping single molecule sequencing reads using basic local alignment with successive refinement (BLASR): application and theory. BMC Bioinformatics 13:238.

11. Chin, C.-S., D.H. Alexander, P. Marks, A.A. Klammer, J. Drake, C. Heiner, A. Clum, A. Copeland, et al. 2013. Nonhybrid, finished microbial genome assemblies from long-read SMRT sequencing data. Nat. Methods 10:563569.

12. Quail, M.A., T.D. Otto, Y. Gu, S.R. Harris, T.F. Skelly, J.A. McQuillan, H.P. Swerdlow, and S.O. Oyola. 2011. Optimal enzymes for amplifying sequencing libraries. Nat. Methods 9:10-11.

13. 15. Wang, M., C.R. Beck, A.C. English, Q. Meng, C. Buhay, Y. Han, H.V. Doddapaneni, F. Yu, et al. 2015. PacBiol.-LITS: a large-insert targeted sequencing method for characterization of human disease-associated chromosomal structural variations. BMC Genomics 16:214.

14. Myers, E.W., G.G. Sutton, A.L. Delcher, I.M. Dew, D.P. Fasulo, M.J. Flanigan, S.A. Kravitz, C.M. Mobarry, et al. 2000. A whole-genome assembly of Drosophila. Science 287:2196-2204.

15. Liang, M., C. Raley, X. Zheng, G. Kutty, E. Gogineni, B.T. Sherman, Q. Sun, X. Chen, et al. 2016. Distinguishing highly similar gene isoforms with a clustering-based bioinformatics analysis of PacBiol. single-molecule long reads. BioData Min. 9:13.

16. Berlin, K., S. Koren, C.-S. Chin, J.P. Drake, J.M. Landolin, and A.M. Phillippy. 2015. Assembling large genomes with single-molecule sequencing and locality-sensitive hashing. Nat. Biotechnol. 33:623-630.

17. Witek, K., F. Jupe, A.I. Witek, D. Baker, M.D. Clark, and J.D.G. Jones. 2016. Accelerated cloning of a potato late blight-resistance gene using RenSeq and SMRT sequencing. Nat. Biotechnol. 34:656-660.

18. Hulbert, S.H., C.A. Webb, S.M. Smith, and Q. Sun. 2001. RESISTANCE GENE COMPLEXES: Evolution and Utilization. Annu. Rev. Phytopathol. 39:285-312.

19. Sun, Q., N.C. Collins, M. Ayliffe, S.M. Smith, J. Drake, T. Pryor, and S.H. Hulbert. 2001. Recombination between paralogues at the Rp1 rust resistance locus in maize. Genetics 158:423438.

20. Heavens, D., G.G. Accinelli, B. Clavijo, and M.D. Clark. 2015. A method to simultaneously construct up to 12 differently sized Illumina Nextera long mate pair libraries with reduced DNA input, time, and cost. Biotechniques 59:42-45.

21. Putnam, N.H., B.L. O'Connell, J.C. Stites, B.J. Rice, M. Blanchette, R. Calef, C.J. Troll, A. Fields, et al. 2016. Chromosome-scale shotgun assembly using an in vitro method for long-range linkage. Genome Res. 26:342-350.

Received 26 April 2016; accepted 06 October 2016

Address correspondence to Matthew D. Clark, Earlham Institute (EI), Norwich Research Park, Norwich, UK, NR4 7UG. E-mail: Matt.Clark@earlham.ac.uk

To purchase reprints of this article, contact: biotechniques@fosterprinting.com 